\title{
Microwaves trigger thermo-acoustic ultrasound generation
}

\author{
Frederic Cegla* \\ Imperial College London, London, England, United Kingdom
}

Ultrasound is one of the most common modalities for diagnostic imaging of the body (biomedical imaging) and engineering structures (non-destructive evaluation-NDE). This is partly due to the lack of safety concerns when probing the body with acoustic waves and the availability and relative affordability of the equipment. Another very important strength of ultrasound is the penetration depth of ultrasonic waves into opaque materials. Frequencies in the low $\mathrm{MHz}$ range can usually penetrate deep inside tissue and other structures. However, ultrasound also has some drawbacks: in particular it usually has poor spatial resolution and requires the use of a range of special frequency band-limited transducers. There is on-going research work that tries to improve the spatial resolution by investigating techniques such as super-resolution algorithms, ${ }^{1}$ micro-bubbles ${ }^{2}$ and photo-acoustic (PA) imaging. ${ }^{3}$

PA imaging is a mixed technique that uses the high spatial resolution of electromagnetic waves in the visible or near visible part of the spectrum and the different absorption coefficient of structures inside tissue to turn the tissue itself into ultrasound sources. As strongly absorbing parts of the tissue heat up more than their surroundings they expand and emit a pressure pulse that travels through the tissue. The pressure pulse is then received by an ultrasonic transducer. This enables the formation of high-resolution images of internal structures that are captured at the outside of the body using ultrasound transducers. This technique is able to produce stunning images; however, it still relies on photons from an external source travelling inside the body and interacting with the tissue. Since the optical absorption of tissue is still relatively high, the penetration depth into the body is limited.

The work of Lan et al. ${ }^{4}$ was inspired by the work on PA and they investigated other means of thermo-acoustic generation of ultrasound. They focused on the use of microwaves, that can penetrate deeper into the tissue. By considering the physics of energy absorption and Poynting's relation of energy conservation, they stipulated that high intensity electric or magnetic fields are required to maximize the thermo-acoustic source strength. They then showed that a split ring resonator (SRR) is an effective way of increasing the magnitude of the electric field in a small spatial area, i.e. where a ring of a wire conductor (roughly $13 \mathrm{~mm}$ diameter, $0.2 \mathrm{~mm}$ cross section) is split. They simulated and built a device that could be shown to induce local heating when it was excited with microwaves at about $2 \mathrm{GHz}$. They demonstrated local temperature increases of almost $70 \mathrm{~K}$ when the SRR was actuated by a microwave of $100 \mathrm{~W}$ power for $0.25 \mathrm{~s}$. Furthermore, they demonstrated that the SRR was an efficient broadband ultrasound source and that it was possible to receive ultrasound signals with SNR $\sim 30$ that were produced when the SRR was placed inside a breast phantom and the microwave had to travel about $50 \mathrm{~mm}$ through the tissue-mimicking phantom.

The work therefore describes an interesting and promising concept to excite ultrasonic waves wirelessly over a relatively large range without any complicated circuitry. Nonetheless, at $\sim 13 \mathrm{~mm}$ diameter the SRR is relatively large and it would be difficult to insert into the body. Therefore, for practical applications, it will be important to see if the concept can be adapted to build microwave resonator-based thermoacoustic ultrasound sources with smaller spatial footprints. It is thus to be expected that the work of Lan et al. will act as a catalyst for further work in the field.

\section{References}

1. C. Fan et al., "A comparison between ultrasonic array beamforming and super resolution imaging algorithms for non-destructive evaluation," Ultrasonics 54(7), 1842-1850 (2014).

2. K. Christensen-Jeffries et al., "Super-resolution ultrasound imaging," Ultrasound Med. Biol. 46(4), 865-891 (2020).

3. M. Xu and L. V. Wang, "Photoacoustic imaging in biomedicine," Rev. Sci. Instrum. 77(4), 041101 (2006).

4. L. Lan et al., "Ultra-efficient thermo-acoustic conversion through a split ring resonator," Adv. Photonics 2(3), 036006 (2020).

Frederic Cegla received his $\mathrm{PhD}$ in mechanical engineering from Imperial College in 2006. He is currently a reader (associate professor) in mechanical engineering at Imperial College with a research interest in physical acoustics and non-destructive evaluation. He also currently chairs the Physical Acoustics Group at the Institute of Physics (IOP).
*Address all correspondence to Frederic Cegla, E-mail: f.cegla@imperial.ac.uk

(C) The Authors. Published by SPIE and CLP under a Creative Commons Attribution 4.0 Unported License. Distribution or reproduction of this work in whole or in part requires full attribution of the original publication, including its DOI. [DOI: 10.1117/1 .AP.2.3.030501] 